\title{
Investigation on glassy skin formation of porous polystyrene fibers electrospun from DMF
}

\author{
M. M. Demir \\ Department of Chemistry, İzmir Institute of Technology, Gülbahçe Köyü, Urla 35430 Izmir, Turkey
}

Received 20 August 2009; accepted in revised form 5 October 2009

\begin{abstract}
Micrometer and submicrometer diameter of polystyrene (PS) fibers were electrospun from various dimethyl formamide (DMF) solutions at different weight fractions under 35\% relative humidity. Increasing polymer fraction in the solution results in a gradual morphological transition from beads-with-incipient to bead-free fibers and also increases the diameter. The formation of uniform glassy skin presumably due to radial capillary flow within the liquid jet was confirmed by scanning electron microscope. The thickness of the skin varies with the weight fraction of PS; therefore, it was normalized with respect to average fiber diameter (AFD). The skin gets thinner as the weight fraction of PS increases. In addition, the fibers exhibit highly porous internal structure and smooth surface along with slight porosity. The development of porosity is attributed to liquid-liquid phase separation of water molecules in atmospheric moisture and DMF.
\end{abstract}

Keywords: nanomaterials, electrospinning, capillary flow, humidity, porosity

\section{Introduction}

Electrospinning has been proven as a robust technique to produce polymeric fibers whose diameter is on the order of a few tens to hundreds of nanometers [1]. In a typical process, a high electrical potential is applied between a polymer solution placed into a reservoir and a grounded conductive target. When the applied electrical force overcomes surface tension of the solution droplet at the nozzle of the reservoir, a charged jet is ejected from the droplet. The jet becomes thinner as it flows through the collector due to both solvent evaporation and continuous electrical force stretching. The polymer concentration gradually increases on the surface of the jet due to solvent loss. This process eventually leaves a dense, skin-like external boundary along the perimeter of the resulting fiber. While it has been accepted that a thin and mechanically distinct skin is formed initially on the charged liquid jet during solidification of fibers [2], experimental results exhibiting the formation of glassy skin on electrospun fibers have not been reported. In this work, we have verified this phenomenon by microscopic observations on electrospun PS fibers and quantified the skin thickness with respect to average fiber diameter (AFD).

A nonwoven fiber mat having a high surface area to volume ratio on the order of $1-100 \mathrm{~m}^{2} / \mathrm{g}$ is achieved by electrospinning, which offers numerous existing and potential applications in filtration [3], catalysis $[4,5]$, and tissue engineering [6]. The surface area of the mats can be further enlarged when surface and/or interior porosity is developed within the electrospun filaments [7, 8]. Two main approaches have been employed to produce porosity in the fibers. One approach is leaching one of the phases out from a bicomponent fiber systems for example polymer blends $[9,10]$ and the fibers remain rough and porous. Using similar methodology, not only polymeric but also porous ceramic fibers can be

*Corresponding author, e-mail: mdemir@iyte.edu.tr

(C) BME-PT 
readily obtained by calcination of polymeric component from binary polymer/metal salt systems [11]. Another approach to produce porosity in electrospun fibers is to use highly volatile co-solvents $[9,12]$. Rapid solvent evaporation causes phase separation into polymer-rich and solvent-rich domains. The evaporation of volatile co-solvents leaves behind voids thereby porosity along the fiber volume is achieved. This approach was extended to electrospinning of hydrophobic polymers dissolved in a water-compatible solvent in a humid environment [13] because the liquid-liquid phase separation can occur even in the presence of small amount of nonsolvent [14], e.g. moisture in air. The charged jet uptakes water from the vapor, which acts as a nonsolvent for the polymers. The miscibility of water with solvent causes formation of liquid-liquid phase separation and finally porous features within the fibers occur. Recently, Pai et al. reported interior porosity of polystyrene fibers prepared from DMF at different levels of humidity and influence of this internal morphology on mechanical strength and stiffness of the electrospun fibers [15]. The same group also pointed out the development of wrinkled surface topography of fibers that result from buckling instability of the charged jet in poly(acrylonitrile) (PAN) and PS. Rabolt et al. investigated the influence of polymer/solvent properties using a variety of solvents on fibers surface morphology [16] and demonstrated the formation of pores on the surface of PS fibers electrospun in humid environment [17]. Here, we electrospun PS from DMF solution at 35\% relative humidity and also demonstrated that the resulting fibers have both interior and surface porosity.

\section{Experimental}

Polystyrene (PS) $\left(M_{n}=170 \mathrm{~kg} / \mathrm{mol} M_{w}=350 \mathrm{~kg} / \mathrm{mol}\right)$ and dimethylformamide (DMF) (ACS reagent, $>99 \%$ ) were provided from Aldrich and Fluka, respectively. They were used without further purification. Four different weight fraction solutions $(0.05,0.10,0.15$, and 0.25$)$ were prepared. These clear solutions were subjected to electrospinning at $3 \mathrm{kV} / \mathrm{cm}$ using a power supply, Gamma Voltage ES $40 \mathrm{P}-20 \mathrm{~W}$. In all experiments, the instrumental parameters were unchanged such that the applied voltage and source-to-target distance were $15 \mathrm{kV}$ and $5 \mathrm{~cm}$, respectively. Flow rate $(\sim 3 \mathrm{~mL} / \mathrm{h})$ was controlled by an infusion pump. The fibers were imaged by scanning electron microscopy (SEM) of Philips XL-30S FEG. The diameter of PS fibers was obtained from SEM images using image J software from at least measurement of 100 polystyrene fibers. In order to examine the internal structure of the fibers, electrospun mats were kept in liquid nitrogen for a while to decrease temperature far below the glass transition temperature of PS and the constituent fibers were broken. The broken end of the fibers was found in electrospun mat by SEM and cross sectional morphology was examined using ultrahigh resolution mode. Two sources of uncertainties may be affecting the observations of skin thickness. First, the SEM images appear to be tilted, therefore the thickness may not be measured properly. To minimize the effect of tilting, the thickness was measured from several points of the skin and the results were averaged out. Second, the border between skin and bulk was not clear especially for the fibers obtained at low polymer fractions. This is because the measured thickness value was normalized with respect to AFD for true comparison.

\section{Results and discussion}

Chain entanglement is one of the important parameters for stability of liquid jet in electrospinning [18, 19]. It is the effect of $\phi_{p}$ and $M$ where $\phi_{p}$ is volume fraction and $M$ is weight average molecular weight. The entanglement can be quantified with entanglement number in solution, $\left(n_{e}\right)_{\text {soln }}=\left(\phi_{p} M_{w} / M_{e}\right)$ using $\rho=1.05 \mathrm{~g} / \mathrm{cm}^{3}$ and $M_{e}=16600 \mathrm{~g} / \mathrm{mol}$. SEM images of PS fibers electrospun from four solutions of different weight fractions are presented in Figure 1. The structure of the fibers varies significantly with the polymer weight fraction. At the lowest fraction (0.05), the charged jet is unstable and bead-withincipient fibers were obtained (panel a). The formation of incipient fibers suggests some degree of chain entanglement in solution, $\left(n_{e}\right)_{\text {soln }}<1$. At 0.10 , beads-on-string morphology is achieved. The solution entanglement number calculated for this condition is $\sim 2$. Increasing the polymer fraction to 0.15 yields continuous structure in which the occurrence of beads diminished gradually and their shape becomes more spindle-like (panel c). At this fraction, chain entanglement number is $\left(n_{e}\right)_{\text {soln }} \sim 3$ and the liquid jet was fully stabilized. Further 


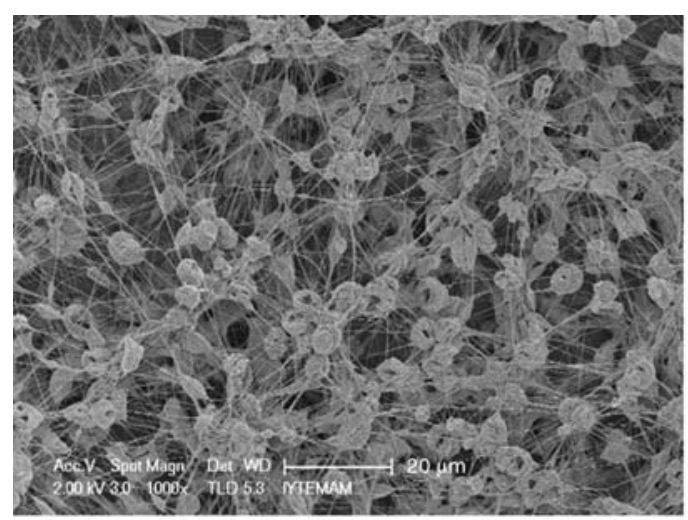

a)

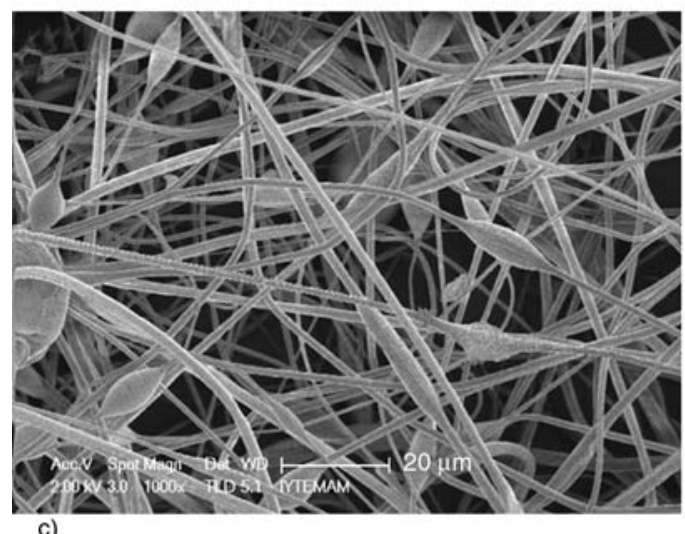

c)

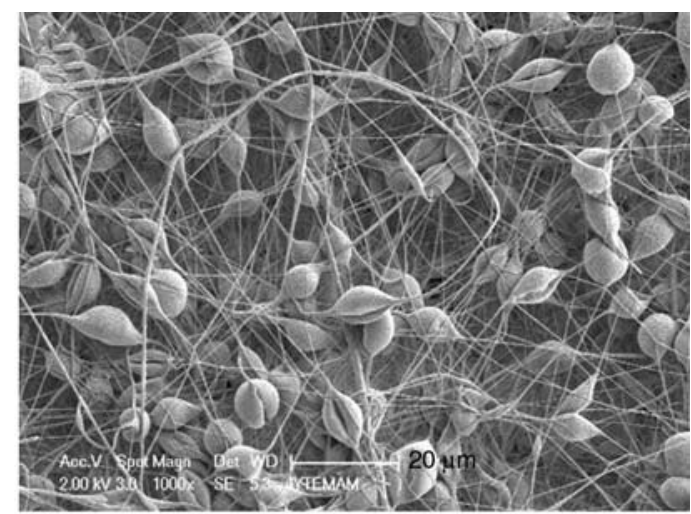

b)

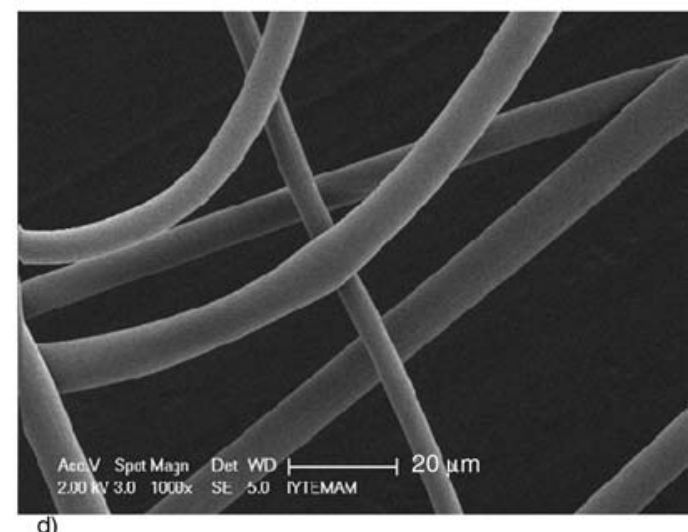

d)

Figure 1. SEM images of PS fibers obtained from DMF solutions at different weight fractions under $35 \%$ relative humidity. (a) 0.05 , (b) 0.10 (c) 0.15 , and (d) 0.25

increase of fraction to 0.25 increases viscosity of solution due to extensive chain entanglements, $\left(n_{e}\right)_{\text {soln }} \sim 4.8$. Hence, continuous and bead-free thick fibers were produced. The general trend of morphological transition presented here is consistent with literature, which was obtained for electrospinning of both PS and some other polymer/solvent systems [18].

The dependences of AFD and skin thickness normalized with respect to AFD on PS weight fraction are demonstrated in Figure 2. A power law relationship was found between AFD and weight fraction such that AFD (weight fraction) $)^{2.4}$. This expression is very close to the one we reported previously for polyurethane urea/DMF system [20]. At the most dilute solution (0.05), the diameter of bead-with-incipient fibers is $\sim 100 \mathrm{~nm}$. An apparent broken end of fiber was not observed since the mat is mainly composed of beads on the order of several $\mu \mathrm{m}$. However, a cross sectional morphology is gradually developed as the polymer fraction in solution increases. Panel $\mathbf{a}, \mathbf{b}$, and $\mathbf{c}$ of Figure 3 show broken end of the fibers obtained from solutions of $0.10,0.15$, and 0.25 , respectively. The skin

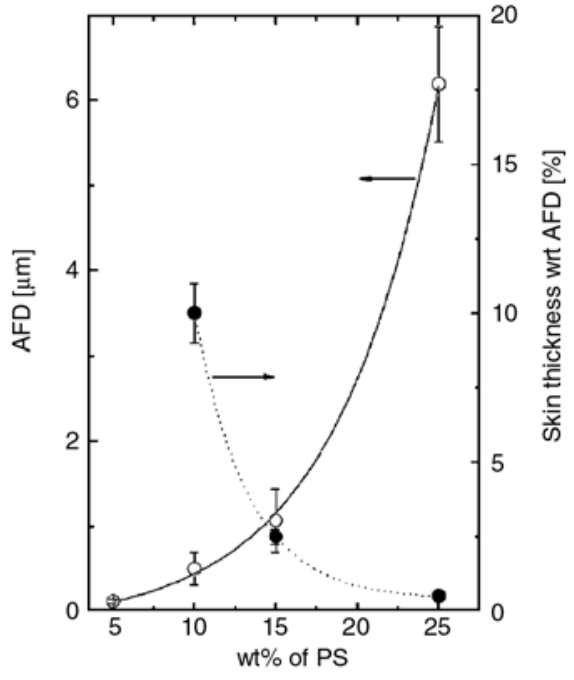

Figure 2. Average fiber diameter (AFD) and skin thickness of fibers normalized with respect to AFD as functions of weight fraction of PS

layer is much thinner than the diameter of fibers and its thickness varies with the fraction of PS in electrospinning solution. To make a true comparison, it was normalized with respect to AFD (right $y$-axis of Figure 2). In contrast to AFD, the normalized skin thickness is inversely proportional with weight fraction of PS. In other words, the skin layer 


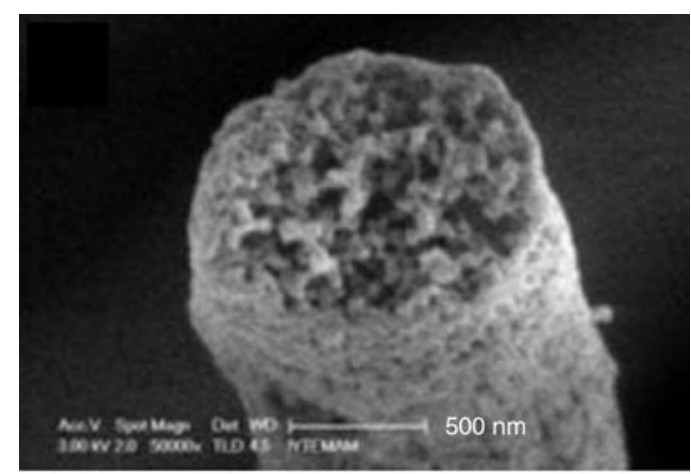

a)

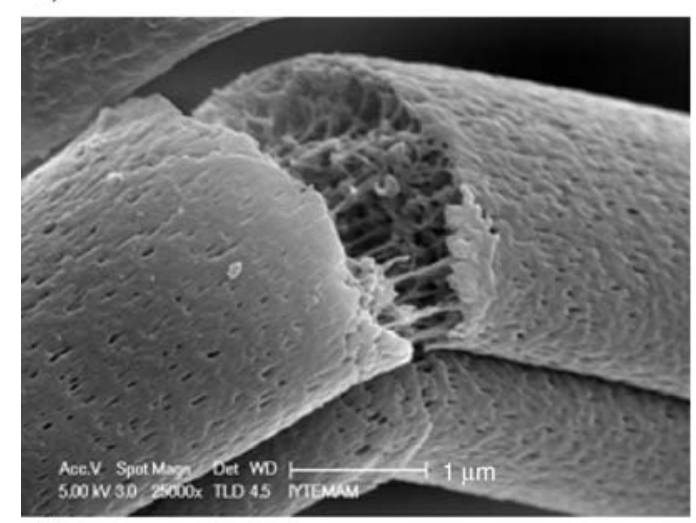

b)

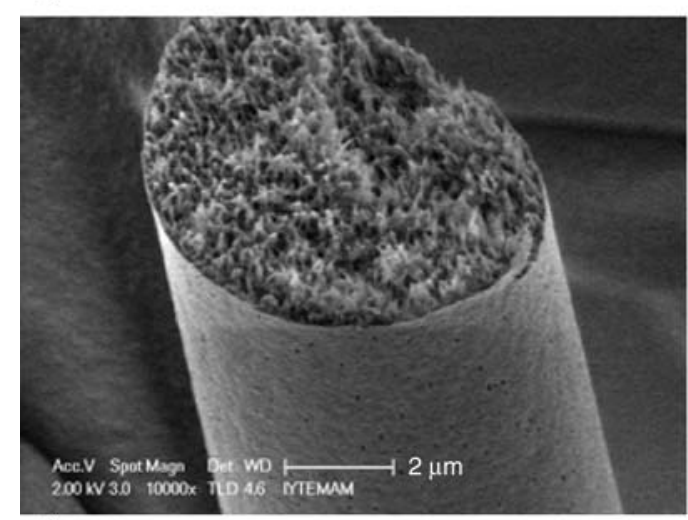

c)

Figure 3. Crossectional profiles of PS fibers electrospun from solutions at (a) 0.10 , (b) 0.15 , and (c) 0.25 weight fraction

gets thinner and sharper as the weight fraction increases.

The characteristic skin formation of electrospun fibers can be attributed to radial capillary flow of solution through the surface of the liquid jet. When a drop of a polymer solution is deposited on a substrate, polymer molecules are concentrated at the edge of the droplet although the molecules are initially homogeneously dispersed over the entire dispersion [21]. Solvent evaporates and the liquid evaporating from the edge is replenished by liquid from the interior. The resulting outward flow can carry the dispersed solid ingredients through the perimeter of the droplet. A ring made up of the ingredients is achieved after complete evaporation of solvent. We have verified this phenomenon for tiny droplet of dilute polystyrene solutions on an atomically flat mica surface [22]. In electrospinning, we propose that polymer molecules concentrate on the external boundary of the liquid jet due to capillary flow and causes characteristic formation of glassy skin during evaporation of solvents. In fact, this phenomenon could take place within the solution droplet at the nozzle of the container prior to jet formation. In addition to capillary effect, charge repulsion within the liquid jet may also contribute this radial flow. In dilute polymer solutions where viscosity is low, the flow deposits polymeric ingredient to the surface of liquid jet. Evaporation of solvent on the outhermost layer leaves behind a dense skin. The solution in the core of these semisolidified fibers appears to be solvent rich. A liquid-liquid phase separation taking place between atmospheric moisture in air and DMF lead to the formation of large solvent rich domains. Complete evaporation of solvent from the core finally imparts large internal voids covered with thick polymer skin with respect to AFD. On the other hand, at high polymer fractions, viscosity of solution increases due to extensive chain entanglements; therefore the effect of evaporative flow does not occur as strongly as in the dilute solution and only limited amount of polymer molecules is carried to the boundary. Rapid solvent evaporation on the surface results in a thin skin layer. For example, the thickness of the layer is around $1 \%$ of the average diameter of the fiber at 0.25 fraction. The presence of low fraction of solvent sets in liquid-liquid separation with smaller domains in the core of the jet. Thus, smaller voids with homogeneous distribution throughout the cross section of the fibers are developed.

Figure 4a shows cross section of polystyrene fibers electrospun from DMF solution at 0.25 . A thin uniform skin surrounding a homogeneously porous core is evident. Even though the fiber core remains robust, the skin of fiber is fractured down as indicated with a white arrow. This observation suggests the brittle and glassy nature of the skin. Figure $4 \mathrm{~b}$ presents higher magnified image of a representative fiber. Two types of pores here can be identified: i) the interior porosity whose size is approximately $100 \mathrm{~nm}$, ii) the surface porosity in the range of 30 $70 \mathrm{~nm}$. Specific surface area of the fibers was found 


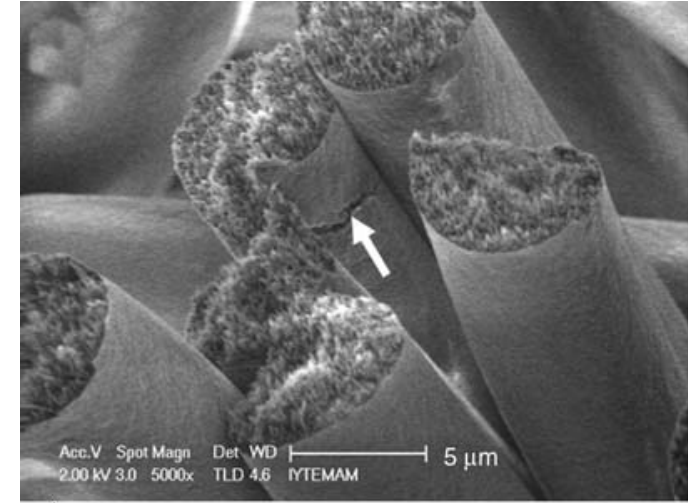

a)

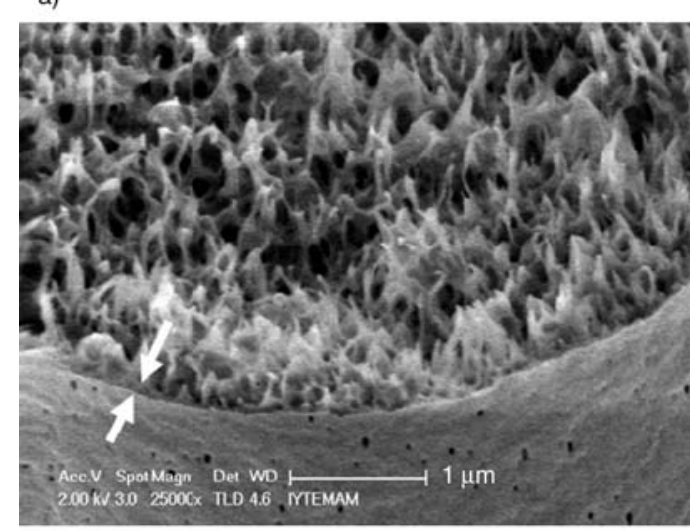

b)

Figure 4. Crossectional SEM images of the electrospun fibers prepared from solution at 0.25 (a) $5000 \times$ and (b) 25 000x

$3.89 \mathrm{~m}^{2} / \mathrm{g}$ by BET nitrogen absorption. This value is more than six times higher than the theoretical surface area of smooth PS fibers $\left(0.62 \mathrm{~m}^{2} / \mathrm{g}\right)$ where the fibers have $6.19 \mu \mathrm{m}$ in diameter on average with no beads. Based on the SEM images, the interior porosity appears to be stronger than the surface porosity. Therefore, the contribution of former porosity to the surface area of entire system is expected to be much higher than that of the latter one.

Although electrospinning process is very fast and polymer molecules are far from equilibrium, this porous morphology may be a consequence of phase separation occurring between moisture in air and DMF. This phenomenon is very well-known and widely discussed in literature [16]. There are two relevant phase separation mechanisms in the course of fiber evolution: thermally induced phase separation (TIPS) and vapor-induced phase separation (VIPS). The former is associated with the rapid solvent evaporation that lowers the local temperature on the liquid jet although the temperature of spin- ning environment is unchanged. The evaporative cooling leads to condensation of moisture in air and the formation of breath figures [23]; therefore, pores occur only on the surface of the fibers. The latter is ascribed to the presence of water vapor in the surrounding air which acts as nonsolvent. The polymer solution undergoes phase separation by diffusion of water vapor. The pore formation is governed by polymer fraction. Increasing fraction of polymer increases the internal pore formation, which is consistent with our results. Since electrospun PS fibers we have produced have both interior and surface porosity, we can conclude that both phase separation mechanisms coexist in formation of PS fibers. However, VIPS appears to have stronger contribution compared to TIPS. It has to be noted that the formation of fused structure or wrinkled surface morphology due to the difference between evaporation rate of water and DMF was not observed.

\section{Conclusions}

The formation of uniform glassy skin on porous electrospun PS fibers was demonstrated. Capillary flow of polymer solution through the external boundary of charged liquid jet may be the main contributing mechanism in skin formation. The thickness of skin normalized with respect to AFD was found to be inversely proportional to the weight fraction of polymer in solution. The electrospun solutions experience absorption of water from humid environment into the polymer/ solvent system. Porosity is induced by phase separation resulting from the rapid evaporation of water molecules from polystyrene/DMF system. The resulting electrospun mats contain three types of pores at different length scales. The first type of pore is the interstitial spaces between the fibers, which is on the order of micrometer. The other two are surface and internal pores of fibers and their size is in nanometer scale. This feature fits with the needs of various applications concerning high surface area such as filtration, catalysts, sensors, membrane and adsorption of living cells for scaffolds in tissue engineering. Similar porous structure possibly occurs for a sort of polymers where DMF and water act as solvent and nonsolvent, respectively. 


\section{Acknowledgements}

The author thanks members of IYTE-MAM for microscope images and BET analysis and M. Çelik for his contribution to the initial stage of the experiments. M.M.D. acknowledges the financial support of The Scientific and Technological Research Council of Turkey (TUBITAK) career development project encoded with TBAG-107T795.

\section{References}

[1] Reneker D. H., Yarin A. L.: Electrospinning jets and polymer nanofibers. Polymer, 49, 2387-2425 (2008). DOI: $10.1016 /$ j.polymer.2008.02.002

[2] Koombhongse S., Liu W. X., Reneker D. H.: Flat polymer ribbons and other shapes by electrospinning. Journal of Polymer Science Part B: Polymer Physics, 39, 2598-2606 (2001). DOI: $10.1002 /$ polb.10015

[3] Yoon K., Kim K., Wang X. F., Fang D. F., Hsiao B. S., Chu B.: High flux ultrafiltration membranes based on electrospun nanofibrous PAN scaffolds and chitosan coating. Polymer, 47, 2434-2441 (2006).

DOI: 10.1016/j.polymer.2006.01.042

[4] Demir M. M., Gulgun M. A., Menceloglu Y. Z., Erman B., Abramchuk S. S., Makhaeva E. E.: Khokhlov A. R., Matveeva V. G., Sulman M. G.: Palladium nanoparticles by electrospinning from poly(acrylonitrile-co-acrylic acid)- $\mathrm{PdCl}_{2}$ solutions. Relations between preparation conditions, particle size, and catalytic activity. Macromolecules, 37, 1787-1792 (2004).

DOI: $10.1021 / \mathrm{ma} 035163 \mathrm{x}$

[5] Demir M. M., Ugur G., Gülgün M. A., Menceloglu Y. Z.: Glycidyl-methacrylate-based electrospun mats and catalytic silver nanoparticles. Macromolecular Chemistry and Physics, 209, 508-515 (2008).

DOI: $\underline{10.1002 / \mathrm{macp} .200700544}$

[6] Nisbet D. R., Forsythe J. S., Shen W., Finkelstein D. I., Horne M. K.: Review paper: A review of the cellular response on electrospun nanofibers for tissue engineering. Journal of Biomaterials Applications, 24, 7-29 (2009). DOI: $10.1177 / 0885328208099086$

[7] Zhang Y. Z., Feng Y., Huang Z-M., Ramakrishna S., Lim C. T.: Fabrication of porous electrospun nanofibres. Nanotechnology, 17, 901-908 (2006). DOI: $10.1088 / 0957-4484 / 17 / 3 / 047$

[8] McCann J. T., Marquez M., Xia Y. N.: Highly porous fibers by electrospinning into a cryogenic liquid. Journal of the American Chemical Society, 128, 14361437 (2006). DOI: $10.1021 / \mathrm{ja} 056810 \mathrm{y}$
[9] Bognitzki M., Frese T., Steinhart M., Greiner A., Wendorff J. H., Schaper A., Hellwig M.: Preparation of fibers with nanoscaled morphologies: Electrospinning of polymer blends. Polymer Engineering and Science, 41, 982-989 (2001).

DOI: 10.1002/pen.10799

[10] Moon S., Choi J., Farris R. J.: Highly porous polyacrylonitrile/polystyrene nanofibers by electrospinning. Fibers and Polymers, 9, 276-280 (2008). DOI: $10.1007 / \mathrm{s} 12221-008-0044-\mathrm{y}$

[11] McCann J. T., Li D., Xia Y. N.: Electrospinning of nanofibers with core-sheath, hollow, or porous structures. Journal of Materials Chemistry, 15, 735-738 (2005). DOI: $10.1039 / \mathrm{b} 415094 \mathrm{e}$

[12] Han S. O., Son W. K., Youk J. H., Lee T. S., Park W. H.: Ultrafine porous fibers electrospun from cellulose triacetate. Materials Letters, 59, 2998-3001 (2005). DOI: 10.1016/j.matlet.2005.05.003

[13] Wang L. F., Pai C-L., Boyce M. C., Rutledge G. C.: Wrinkled surface topographies of electrospun polymer fibers. Applied Physics Letters, 94, 151916/1151916/3 (2009). DOI: $\underline{10.1063 / 1.3118526}$

[14] Matsuyama H., Teramoto M., Nakatani R., Maki T.: Membrane formation via phase separation induced by penetration of nonsolvent from vapor phase. I. Phase diagram and mass transfer process. Journal of Applied Polymer Science, 74, 159-170 (1999).

[15] Pai C-L., Boyce M. C., Rutledge G. C.: Morphology of porous and wrinkled fibers of polystyrene electrospun from dimethylformamide. Macromolecules, 42, 2102-2114 (2009).

DOI: $10.1021 / \mathrm{ma} 802529 \mathrm{~h}$

[16] Megelski S., Stephens J. S., Chase D. B., Rabolt J. F.: Micro- and nanostructured surface morphology on electrospun polymer fibers. Macromolecules, 35, 8456-8466 (2002).

DOI: $10.1021 / \mathrm{ma} 020444 \mathrm{a}$

[17] Casper C. L., Stephens J. S., Tassi N. G., Chase D. B., Rabolt J. F.: Controlling surface morphology of electrospun polystyrene fibers: Effect of humidity and molecular weight in the electrospinning process. Macromolecules, 37, 573-578 (2004).

DOI: $\underline{10.1021 / \mathrm{ma} 0351975}$

[18] Shenoy S. L., Bates W. D., Frisch H. L., Wnek G. E.: Role of chain entanglements on fiber formation during electrospinning of polymer solutions: Good solvent, non-specific polymer-polymer interaction limit. Polymer, 46, 3372-3384 (2005).

DOI: 10.1016/j.polymer.2005.03.011

[19] Wang C., Hsu C-H., Lin J-H.: Scaling laws in electrospinning of polystyrene solutions. Macromolecules, 39, 7662-7672 (2006).

DOI: $\underline{10.1021 / \mathrm{ma} 060866 \mathrm{a}}$ 
[20] Demir M. M., Yilgor I., Yilgor E., Erman B.: Electrospinning of polyurethane fibers. Polymer, 43, 33033309 (2002).

DOI: $10.1016 / \mathrm{S} 0032-3861(02) 00136-2$

[21] Deegan R. D., Bakajin O., Dupont T. F., Huber G., Nagel S. R., Witten T. A.: Capillary flow as the cause of ring stains from dried liquid drops. Nature, 389, 827-829 (1997).

DOI: $\underline{10.1038 / 39827}$
[22] Demir M. M., Erman B.: Dimensions of polystyrene particles deposited on mica from dilute cyclohexane solution at different temperatures. Macromolecules, 35, 7986-7992 (2002).

DOI: $10.1021 / \mathrm{ma} 020676+$

[23] Srinivasarao M., Collings D., Philips A., Patel S.: Three-dimensionally ordered array of air bubbles in a polymer film. Science, 292, 79-83 (2001). DOI: $\underline{10.1126 / \text { science. } 1057887}$ 